\title{
Hippocampus-Dependent Memory Formation: Do Memory Type-Specific Mechanisms Exist?
}

\author{
Keiko Mizuno ${ }^{1}$ and Karl Peter Giese ${ }^{1, *}$ \\ ${ }^{1}$ Wolfson Institute for Biomedical Research, University College London, London, WC1E6BT, UK
}

Received April 13, 2005

\begin{abstract}
Long-term memory (LTM) formation requires gene transcription and de novo protein synthesis. The transcription factor CREB is required for hippocampus-dependent LTM formation, and it is activated by several signaling pathways, including protein kinase A (PKA), the mitogen activated protein/extracellular signal-regulated kinases (MAPK or ERKs), and $\mathrm{Ca}^{2+} /$ calmodulin kinases (CaMKs). However, it is unknown whether all types of hippocampusdependent LTM use the same signaling to activate transcription, and whether the transcriptional output is the same. Here we present molecular genetic and behavioral studies to demonstrate that two types of hippocampus-dependent LTM formation, spatial and contextual, require different signaling molecules. This can be illustrated by the CaMK kinases, CaMKK $\alpha$, and $\operatorname{CaMKK} \beta$, which have converse roles. CaMKK $\alpha$ is required for contextual and $\mathrm{CaMKK} \beta$ is required for spatial LTM formation. This leads to the surprising conclusion that there are distinct types of hippocampus-dependent LTM, which differ in their underlying molecular mechanisms.
\end{abstract}

Keywords: hippocampus, cAMP-responsive element binding protein, signaling, $\mathrm{Ca}^{2+} /$ calmodulin kinase, mouse genetics

\section{Introduction}

The study of patient H.M. in the mid 1950s showed that the formation of long-term memory (LTM) requires the medial temporal lobe (brain areas including the hippocampus, the amygdala, and part of the temporal cortex). H.M. lost his ability to generate new LTM after bilateral removal of the medial temporal lobe for treatment of his severe seizures. Even though H.M. lost his ability to generate new LTM, he kept his older memories from before surgery, and he was able to have short-term memory (STM). STM lasts for only a few minutes, whereas LTM can last for days, weeks, or years. In humans, LTM can be distinguished as explicit (declarative) or implicit (non-declarative) memory. Explicit memory is recalled by a deliberate and conscious effort, such as factual knowledge of people, places, and things. Implicit memory is involved in training, reflexive, motor, and perceptual skills. The information from H.M.

*Corresponding author. FAX: +44 2079165994

E-mail: p.giese@ucl.ac.uk

Invited article and other similar patients have demonstrated an essential role of the hippocampus in the formation of explicit but not implicit memory. For example, H.M. can not remember new faces and places, but he can learn new motor skills (1). In rodents lesions of the hippocampus impair memory for space and context, and the hippocampus is thought to be important for creating spatial representations in the brain (2).

Using genetically modified mice, cellular and molecular mechanisms underlying hippocampus-dependent LTM formation have been studied intensively. Because some genes such as $N$-methyl-D-aspartate (NMDA) receptors and the transcription factor cAMP-responsive element binding protein (CREB) are needed for development and survival, traditional null mutant mice are not always suitable for studying mechanisms of LTM formation. Therefore, some mutant mouse lines were generated using the forebrain-specific $\alpha$ CaMKIIpromoter for spatial restriction, and occasionally, the tetracycline-regulated system for temporal reversibility in the expression of dominant-negative transgenes $(3,4)$. Region-restricted deletions of genes, such as in CA1NR1-null mutant mice, were also generated using the 
Temporal control by the tTA system

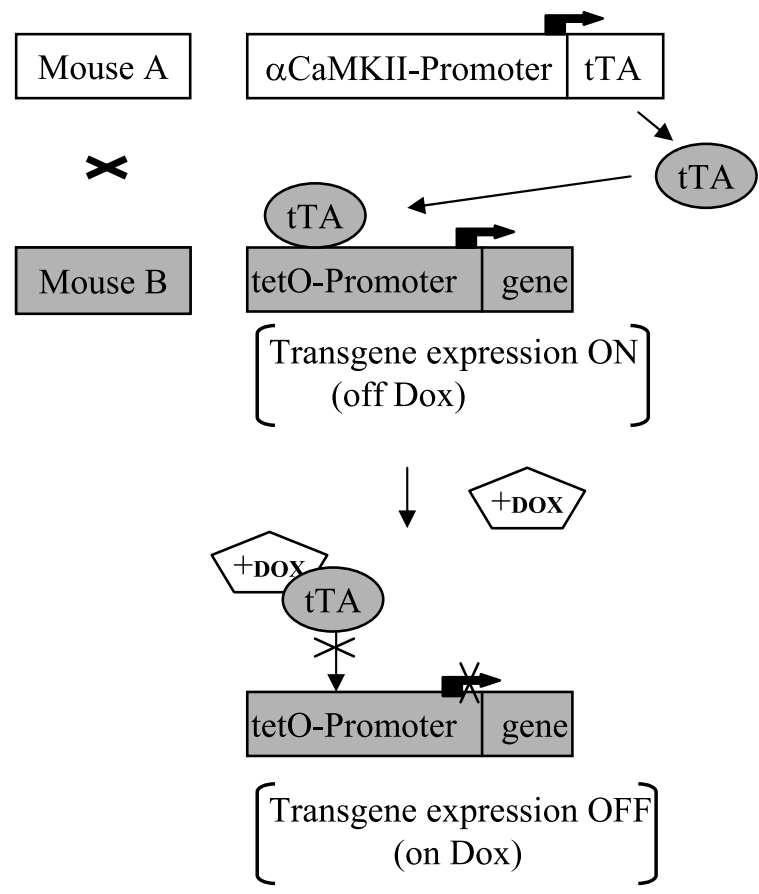

\section{Spatial restriction by the Cre/loxP system}

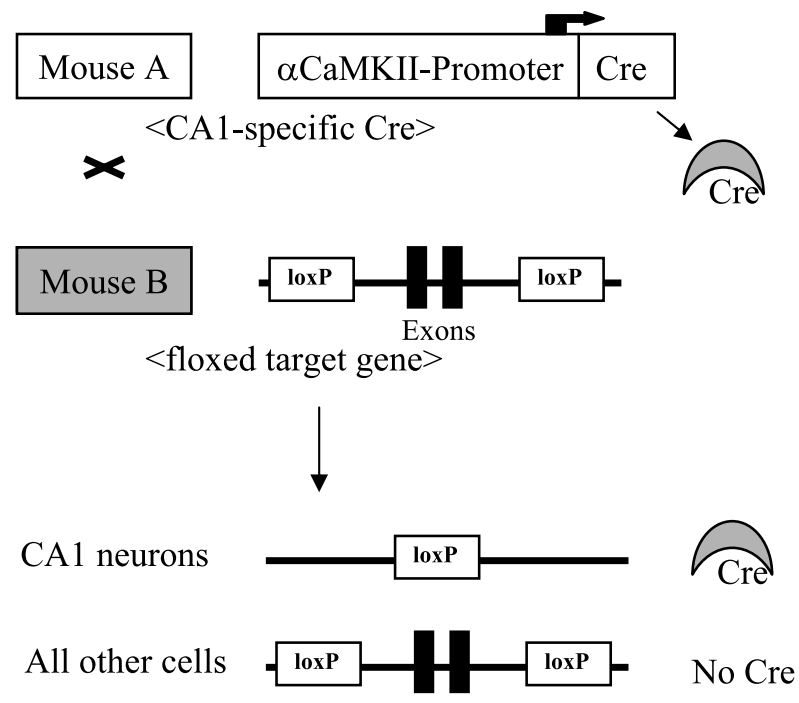

Fig. 1. Genetic techniques to restrict mutations. Left: The tetracycline-dependent regulatory system is used for inducible expression of a transgene. The tetracycline transactivator (tTA) is used as a switch ON/OFF transcription to drive the expression of a gene of interest. A mouse line (Mouse A) that expresses tTA protein under a tissue-type specific promoter $(\alpha \mathrm{CaMKII})$, and a mouse line (Mouse B) that expresses a gene of interest placed downstream of the tet operator (tetO) promoter are intercrossed. The tTA protein binds to the tetO promoter and induces transcription. Whenever the tetracycline (doxycyclin DOX) is present in either drinking water or foods, it blocks transcription. Right: Spatial restriction by the Cre/loxP system is a widely used technique. Cre is a site-specific DNA recombinase derived from the P1 bacteriophage and recognizes loxP sites. Cre catalyzes the deletion of DNA that is flanked by a pair of loxP sites (floxed), creating a null mutation of the gene of interest. A mouse line (Mouse A) that expresses Cre driven by a tissue-specific promoter ( $\alpha$ CaMKII) and a mouse line (Mouse B) that contains the floxed gene are intercrossed. Restricted expression of Cre such as expression only in the hippocampal area CA1 or CA3 leads to a region-restricted deletion of the floxed gene.

phage P1-derived, Cre/loxP recombination system (Fig. 1).

Recently, the molecular genetic analysis of hippocampus-dependent LTM has shown that there are distinct types, which require different signaling molecules. Here we will review this evidence and focus on studies that tested particular mutant mouse lines in both the water maze and contextual conditioning and found impaired LTM formation.

\section{Different types of hippocampus-dependent behav- ioral tasks}

Several hippocampus-dependent LTM tasks exist, but the water maze and contextual fear conditioning are the most commonly used tasks in the field of mouse molecular genetics.

1) Hippocampus-dependent spatial memory forma- tion is frequently investigated in the hidden-platform version of the water maze (5). In the water maze, mice learn to navigate to a submerged platform by using distal, spatial cues in the room. Mice are trained in serial sessions and at the end of training, spatial memory formation is assessed in a probe trial where the platform is removed from the pool. If the mouse has formed normal spatial memory, it will spend significant time in the target quadrant where the platform was located. As a control for performance deficits, a visible platform version of this task is used, which does not require the hippocampus. The water maze is a multi-trial task in which mice learn slowly. It is impossible to distinguish between impairment in learning versus memory with the exception of a few particular LTM impairments (e.g., 6); and furthermore over-training, depending on the training protocol, could potentially miss the detection of an abnormal phenotype. 
2) Another frequently used task, contextual conditioning, is also hippocampus-dependent (7). In this task, mice can learn a new environment (training box) as a whole context, not as context elements. In contextual conditioning, a mouse is exposed to a novel environment, which is the context. A mild foot shock is provided and consequently the mouse associates the context with the shock. When placed back into the context, the mouse remembers the context-shock association and exhibits 'freezing'. Freezing is scored at $1-2 \mathrm{~h}$ (protein synthesis-independent, STM) and $24 \mathrm{~h}$ or longer time points (protein synthesis-dependent, LTM) after the training. Contextual conditioning with a tone presentation requires the dorsal hippocampus and amygdala and also permits testing for amygdala-dependent tone fear conditioning (8). If a particular mutant is impaired in both contextual and tone conditioning tests, it is impossible to establish whether the impaired contextual memory formation resulted from dysfunction of either the hippocampus or the amygdala. Another point to consider is that in some mutants, contextual conditioning may be normal even in the presence of hippocampal dysfunctions because non-hippocampal systems can support contextual conditioning (7). In this case, context discrimination, a more difficult task (9), may be more sensitive and therefore detect impairments in hippocampus-dependent memory formation. On the other hand, the contextual discrimination task may test for another hippocampus-dependent memory type.

\section{Molecular mechanisms of LTM formation}

The process of LTM formation requires gene transcription and de novo protein synthesis in contrast to STM, which requires post-translational modification of proteins (10). The transcription factor CREB is required for hippocampus-dependent LTM formation (11); and signaling by protein kinase A (PKA), the mitogen activated protein/extracellular signal-regulated kinases (MAPK/ERKs), and $\mathrm{Ca}^{2+} /$ calmodulin kinases (CaMKs) have been implicated in activation of CREB, by phosphorylating Serine 133. The transcriptional co-activator CREB-binding protein (CBP) also needs to be phosphorylated to activate CREB-mediated transcription $(11,12)$. CBP plays a role in cognitive function not only as a platform protein, but also as a chromatin remodelling histone acetyltransferase (13). The current view of LTM formation in the hippocampus involves an activation of CREB and CBP. However, it is unknown whether all types of hippocampus-dependent LTM use the same signaling to activate transcription and whether the transcriptional output is the same.

\section{Common signaling for hippocampus-dependent spatial and contextual LTM formation}

Several molecular genetic studies have shown that there is common signaling between spatial and contextual LTM formation.

CREB was the first transcription factor to be studied intensively in LTM formation. CREB hypomorphic mutant mice have impairments in both spatial and contextual LTM formation (14). However, even though these mutants lack expression of the predominant $\alpha$ - and $\delta$-isoforms of CREB, they have a compensatory upregulation in CREB $\beta$ and the cAMP-responsive element modulator (CREM) (15). Additional mutants with inducible expression of a dominant inhibitor of all CREB/ATF transcription factors confirmed a role for CREB in LTM formation $(16,17)$. Although CREB is important for LTM formation, the phenotypes of the CREB hypomorphic mutants and some other CREBspecific transgenic manipulations are inconsistent. We believe that this is due to the large number of CREB/ATF transcription factors including CREM and ATF-1 being expressed in the hippocampus. There is a tight regulation between these transcription factors, probably depending on the genetic background, which influences the behavioral phenotype.

Among the CREB-activating kinases, some $\mathrm{Ca}^{2+} /$ calmodulin-dependent protein kinases (CaMKs) directly connect $\mathrm{Ca}^{2+}$-signaling and gene expression $(12,18)$. One of these kinases is CaMKIV, which phosphorylates CREB, and has a relatively well-studied role in hippocampal LTM. Forebrain-restricted dominant-negative CaMKIV mutant mice (dnCaMKIV) are impaired in spatial and contextual memory formation (19). Contextual memory is impaired 7 days, but not 1 day after conditioning, and the impairment in contextual LTM formation does not seem to be caused by amygdala dysfunction. This is because memory of cued conditioning is not affected. In contrast with these findings, CaMKIV null mutant mice have been described as normal in spatial memory formation (20). However, the testing conditions may not have been sufficiently sensitive to detect an abnormal phenotype. The CaMKIV null mutants are impaired in contextual memory formation, but this impairment is likely to have resulted from amygdala dysfunction because the mutants are also affected in cued conditioning (21).

The neuronal response to a $\mathrm{Ca}^{2+}$ stimulus is a complex process involving direct $\mathrm{Ca}^{2+} /$ calmodulin $(\mathrm{CaM})$ actions as well as secondary activation of multiple signaling pathways such as cAMP and ERK $(12,18)$. Transgenic mice, expressing a dominant inhibitor of CaM selectively in the nuclei of adult forebrain neurons, are 
impaired in contextual memory 1 day but not $1 \mathrm{~h}$ after conditioning LTM formation (22). These mutants are also impaired in spatial memory formation.

The MAP kinase pathway contains at least three protein kinases: the extracellular signal-regulated kinases 1/2 (ERK1 and ERK2), the immediate upstream of the MAP/ERK kinase (MEK or MKK), and the Raf family as MEKK (23). Conditional transgenic mice expressing dominant-negative MEK1 in the postnatal forebrain are impaired in contextual memory 1 day but not $1 \mathrm{~h}$ after conditioning LTM formation (24). These mutants are also impaired in spatial memory formation.

The cAMP-PKA pathway is involved in CREB activation. Dominant-negative PKA mutants have impaired LTM but normal STM in contextual and cued conditioning, and impaired spatial memory formation (25).

The role of c-Fos, an immediate-early gene and a target gene of CREB, was studied in conventional c-fos null mutants, but these mice suffer from developmental malformations (26) and are not suitable for studying learning and memory. A CNS-specific c-fos null mutant (c-fos $\delta^{\mathrm{CNS}}$ ) is impaired in both spatial and contextual LTM formation (27). However, interestingly when the fos-related antigen 1 (Fra-1), a member of c-fos family of transcription factors, was knocked-in into the c-fos locus, it can only replace the function of contextual, but not spatial memory formation (28). This suggests that there are different molecular requirements for spatial and contextual LTM formation.

\section{Molecules required for either spatial or contextual LTM}

Recent findings show that spatial and contextual LTM require for their formation different signaling to regulate gene transcription and they need distinct transcriptional outputs. The $\mathrm{Ca}^{2+} /$ calmodulin kinase kinases, $\mathrm{CaMKK} \alpha$ and $\mathrm{CaMKK} \beta$, can both phosphorylate CaMKIV and this phoshorylation event enhances CaMKIV activity (18). Studies with CaMKK $\beta$ null mutants revealed that this kinase is required for the activation of CREB by spatial training as well as spatial memory formation in the water maze in male mice (29). However, CaMKK $\beta$ is not needed for contextual LTM formation. Thus, for spatial but not contextual LTM formation, CaMKK $\beta$ may activate CaMKIV to phosphorylate CREB. In contrast to $\mathrm{CaMKK} \beta, \mathrm{CaMKK} \alpha$ is required for contextual but not spatial LTM in male mice (30). The requirement for contextual LTM is hippocampus-dependent because $\mathrm{CaMKK} \alpha$ is not needed for cued conditioning. It remains to be investigated whether CaMKK $\alpha$ activates CaMKIV to phosphorylate CREB during contextual but not spatial LTM formation. The studies with $\mathrm{CaMKK} \alpha$ and $\mathrm{CaMKK} \beta$ mutants show that there is a double dissociation for the signaling mechanisms required for spatial and contextual LTM formation. This dissociation between spatial and contextual LTM formation has also been observed in several other mutant mouse lines.

Earlier studies of CREB in LTM formation focused only on CREB. However, CREB can heterodimerize with the other CREB family transcription factors CREM and ATF1, and their functions are not restricted to hippocampus-dependent memory formation (12). To overcome these difficulties, a transgenic mouse line expressing KCREB was generated. KCREB is a mutant of human CREB that is a potent dominant-negative inhibitor, and importantly, it also inhibits all three CREB family transcription factors. dCA1-KCREB mice have restricted expression of KCREB in area CA1 of the dorsal hippocampus (17). These mutants are impaired in spatial LTM formation, but have normal contextual LTM. Importantly, when the KCREB gene is switched off, spatial LTM impairment is rescued. Furthermore, dorsally restricted dCA1-KCREB provided evidences that the dorsal hippocampus is needed for spatial LTM, but not contextual LTM. These results support previous lesion studies that total hippocampal lesions disrupt both the water maze and contextual fear conditioning, whereas ventral lesions disrupt fear conditioning while sparing performance in the water maze (31), and dorsal hippocampal lesions disrupt performance in the water maze only (32). A similar phenotype, impaired spatial and normal contextual LTM, was observed in brainspecific CREB null mutants, which have up-regulated CREM expression (33).

CBP is an important factor to form LTM and functions as a transcriptional co-activator as well as histone acetyltransferase (HAT), which can remodel the chromatin structure. Dominant-negative CBP transgenic mice that specifically lack HAT activity (CBP $\{\mathrm{HAT}-\})$ are impaired in spatial memory formation but they are normal in contextual LTM (34).

As one of the mechanisms to stimulate ERK, the PKA-dependent activation of the Ras-related small G protein Rap1 and B-Raf were identified. Transgenic mice expressing a temporally controlled dominantnegative Rap1 (iRap1) in the forebrain showed that Rap1 is required for spatial memory formation (35). Mice with iRap1 exhibit deficits in contextual discrimination but not in contextual and cued fear conditioning tasks.

The pituitary adenylate cyclase activating polypeptide (PACAP) type I receptor (PAC1) is a G-protein coupled receptor. Null mutants as well as forebrain-specific mutants both have a deficit in contextual but not cued 
fear conditioning, but normal spatial LTM (36).

Brain-derived neurotrophic factor (BDNF) is a target gene of CREB (37). BDNF expression is regulated in the hippocampus after spatial training as well as contextual conditioning $(38,39)$. Since BDNF and its receptor TrkB are expressed throughout the brain from development, it is difficult to know the function specifically in learning and memory using traditional null mutants. To study the specific role of BDNF, forebrain-restricted BDNF null mutants (Emx-BDNFKO mice) were generated (40). Emx-BDNFKO mice are impaired in spatial memory formation but they are normal in contextual LTM. However, in these BDNF mutants, contextual freezing was enhanced in comparison to WT mice, and the mutants generalize their context-shock association to other contexts. Thus, there is the possibility that contextual learning in mutant mice is not hippocampusdependent. Indeed pre-training lesions suggest that "contextual conditioning" can occur without the hippocampus, possibly resulting from discrete cue-shock associations (9).

\section{Conclusion}

The transcription factor CREB was recognised as a molecular switch for LTM formation in the hippocampus. Original findings suggested that CREB is required for LTM formation in general. However, some difficulties were encountered in these earlier experiments to elucidate the detailed roles of CREB in different types of LTM formation. Using region-specific mutations, recent results suggest that not only CREB but also other transcription factors might be important for LTM formation. CREB is more involved in spatial LTM formation, but perhaps other transcription factors are required for contextual LTM formation. For example, c-Rel, a transcription factor of the nuclear factor $\kappa \mathrm{B}$ family, is required in contextual LTM formation (41). Further supporting this idea, we found that CREB activation was regulated during spatial LTM formation and required $\mathrm{CaMKK} \beta$ (29), but we failed to detect any CREB activation during contextual LTM formation (our unpublished data). This might suggest

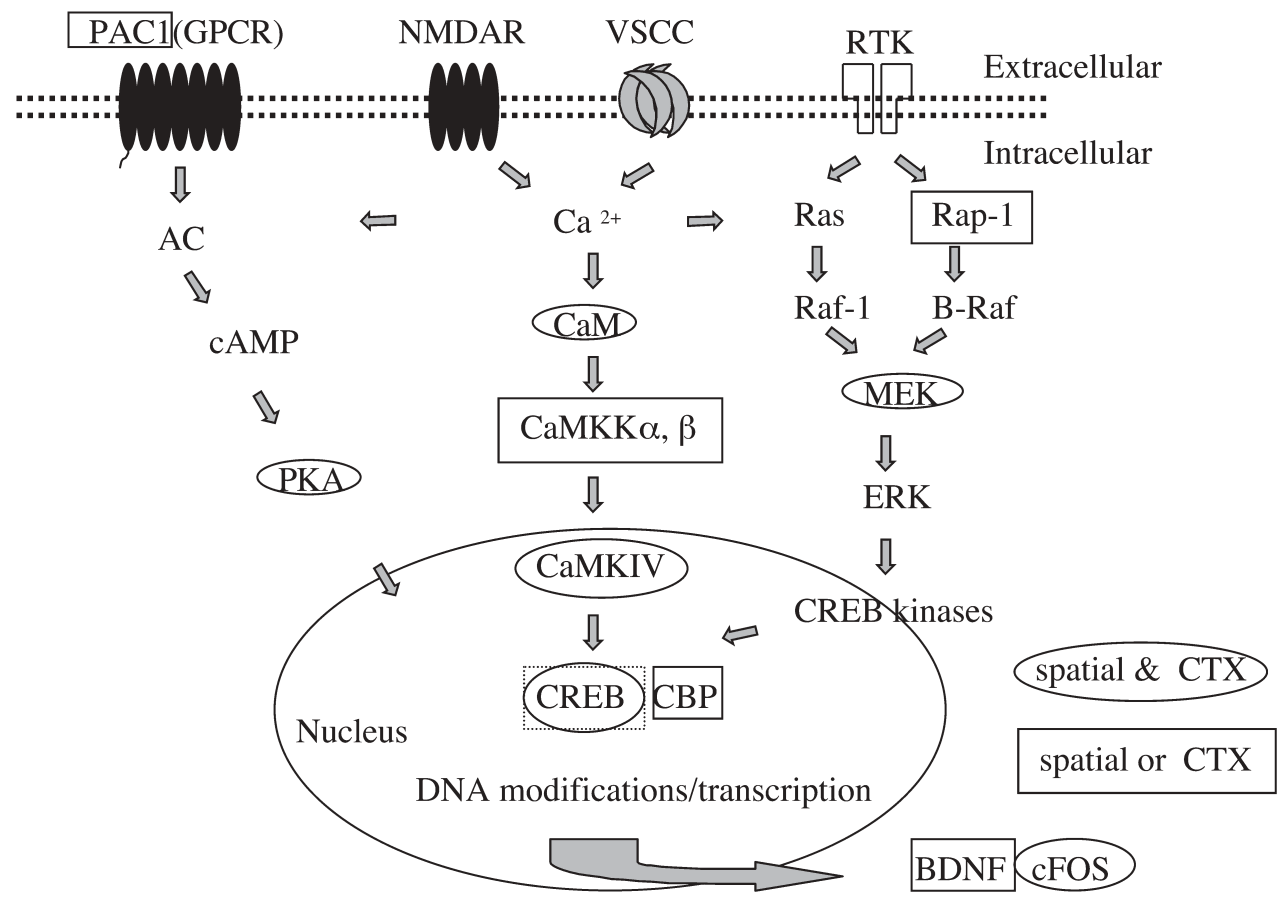

Fig. 2. Summary of the signaling pathways involved in spatial and/or contextual long-term memory (LTM) formation discussed in this review. Excitatory neurotransmitters, ligands for GPCRs, and neuronal growth factors are among the stimuli that activate signaling pathways during hippocampus-dependent LTM formation. It is thought that these signals activate CREB kinases and CREB. Stimulus-dependent kinases include PKA, CaMKIV, and downstream-kinases of ERK such as pp90RSK (RSK), and MSK families of protein kinases are CREB kinases. Some signaling components are required for both spatial and contextual LTM formation (circled) and others are required for either spatial or contextual LTM formation (boxed). Abbreviations: AC: adenylate cyclase, BDNF: brain-derived neurotrophic factor, $\mathrm{CaM}: \mathrm{Ca}^{2+} /$ calmodulin, $\mathrm{CaMKK}: \mathrm{Ca}^{2+} / \mathrm{calmodulin}^{2}$ kinase kinase, CBP: CREB binding protein, CTX: contextual LTM formation, ERK: extracellular signal-regulated kinase, GPCR: G protein-couple receptor, MEK: MAP/ERK kinase, NMDAR: $N$-methyl-D-aspartate receptor, PAC1: PACAP type I receptor, PKA: cAMP-dependent protein kinase, RTK: receptor tyrosine kinase, VSCC: voltage-sensitive calcium channels. 
that contextual LTM formation requires another transcription factor(s) and CaMKK $\alpha$.

We have discussed the signaling molecules whose roles in spatial and contextual LTM formation has been tested using genetically modified mice and this is summarised in Fig. 2. It seems that molecules upstream of CREB are differentially required for distinct types of hippocampus-dependent LTM; CaMKK $\beta$ and Rap1 are involved in spatial, whereas $\mathrm{CaMKK} \alpha$ and PAC1 are needed for in contextual LTM formation. Therefore, we conclude that the formation of two types of hippocampus-dependent LTM, spatial and contextual, uses different signaling molecules and perhaps distinct transcription factors.

\section{Acknowledgments}

We thank L. Drinkwater for helpful comments and are grateful for the generous support from the Wellcome Trust and British Medical Research Council.

\section{References}

1 Milner B, Squire LR, Kandel ER. Cognitive neuroscience and the study of memory. Neuron. 1998;20:445-468.

2 O'Keefe J, Nadel L. The hippocampus as a cognitive map. Oxford: Clarendon Press; 1978.

3 Mayford M, Kandel ER. Genetic approaches to memory storage. Trends Genet. 1999;15:463-470.

4 Nakazawa K, McHugh TJ, Wilson MA, Tonegawa, S. NMDA receptors, place cells and hippocampal spatial memory. Nat Rev Neurosci. 2004;5:361-372.

5 Morris RG, Garrud P, Rawlins JN, O'Keefe J. Place navigation impaired in rats with hippocampal lesions. Nature. 1982;297: 681-683.

6 Remondes M, Schuman EM. Role for a cortical input to hippocampal area CA1 in the consolidation of a long-term memory. Nature. 2004;431:699-703.

7 Anagnostaras SG, Gale GD, Fanselow MS. Hippocampus and contextual fear conditioning: recent controversies and advances. Hippocampus. 2001;11:8-17.

8 Phillips RG, LeDoux JE. Lesions of the dorsal hippocampal formation interfere with background but not foreground contextual fear conditioning. Learn Mem. 1994;1:34-44.

9 Frankland PW, Cestari V, Filipkowski RK, McDonald RJ, Silva AJ. The dorsal hippocampus is essential for context discrimination but not for contextual conditioning. Behav Neurosci. 1998;112:863-874.

10 Silva AJ, Giese KP. Plastic genes are in! Curr Opin Neurobiol. 1994;4:413-420.

11 Silva AJ, Kogan JH, Frankland PW, Kida S. CREB and memory. Annu Rev Neurosci. 1998;21:127-148.

12 Lonze BE, Ginty DD. Function and regulation of CREB family transcription factors in the nervous system. Neuron. 2002;35: 605-623.

13 Levenson JM, Sweatt JD. Epigenetic mechanisms in memory formation. Nat Rev Neurosci. 2005;6:108-118.
14 Bourtchuladze R, Frenguelli B, Blendy J, Cioffi D, Schutz G, Silva AJ. Deficient long-term memory in mice with a targeted mutation of the cAMP-responsive element-binding protein. Cell. 1994;79:59-68.

15 Hummler E, Cole TJ, Blendy JA, Ganss R, Aguzzi A, Schmid $\mathrm{W}$, et al. Targeted mutation of the CREB gene: compensation within the CREB/ATF family of transcription factors. Proc Natl Acad Sci USA. 1994;91:5647-5651.

16 Kida S, Josselyn SA, de Ortiz SP, Kogan JH, Chevere I, Masushige S, et al. CREB required for the stability of new and reactivated fear memories. Nat Neurosci. 2002;5:348-355.

17 Pittenger C, Huang YY, Paletzki RF, Bourtchouladze R, Scanlin $\mathrm{H}$, Vronskaya S, et al. Reversible inhibition of CREB/ATF transcription factors in region CA1 of the dorsal hippocampus disrupts hippocampus-dependent spatial memory. Neuron. 2002; 34:447-462.

18 Soderling TR. The $\mathrm{Ca}^{2+}$-calmodulin-dependent protein kinase cascade. Trends Biochem Sci. 1999;24:232-236.

19 Kang H, Sun LD, Atkins CM, Soderling TR, Wilson MA, Tonegawa S. An important role of neural activity-dependent CaMKIV signaling in the consolidation of long-term memory. Cell. 2001;106:771-783.

20 Ho N, Liauw JA, Blaeser F, Wei F, Hanissian S, Muglia LM, et al. Impaired synaptic plasticity and cAMP response elementbinding protein activation in $\mathrm{Ca}^{2+} /$ calmodulin-dependent protein kinase type IV/Gr-deficient mice. J Neurosci. 2000;20:64596472.

21 Wei F, Qiu CS, Liauw J, Robinson DA, Ho N, Chatila T, et al. Calcium calmodulin-dependent protein kinase IV is required for fear memory. Nat Neurosci. 2002;5:573-579.

22 Limback-Stokin K, Korzus E, Nagaoka-Yasuda R, Mayford M. Nuclear calcium/calmodulin regulates memory consolidation. J Neurosci. 2004;24:10858-10867.

23 Sweatt JD. Mitogen-activated protein kinases in synaptic plasticity and memory. Curr Opin Neurobiol. 2004;14:311-317.

24 Kelleher RJ 3rd, Govindarajan A, Jung HY, Kang H, Tonegawa S. Translational control by MAPK signaling in long-term synaptic plasticity and memory. Cell. 2004;116:467-479.

25 Abel T, Nguyen PV, Barad M, Deuel TA, Kandel ER, Bourtchouladze R. Genetic demonstration of a role for PKA in the late phase of LTP and in hippocampus-based long-term memory. Cell. 1997;88:615-626.

26 Johnson RS, Spiegelman BM, Papaioannou V. Pleiotropic effects of a null mutation in the c-fos proto-oncogene. Cell. 1992;71:577-586.

27 Fleischmann A, Hvalby O, Jensen V, Strekalova T, Zacher C, Layer LE, et al. Impaired long-term memory and NR2A-type NMDA receptor-dependent synaptic plasticity in mice lacking c-Fos in the CNS. J Neurosci. 2003;23:9116-9122.

28 Gass P, Fleischmann A, Hvalby O, Jensen V, Zacher C, Strekalova $\mathrm{T}$, et al. Mice with a fra- 1 knock-in into the c-fos locus show impaired spatial but regular contextual learning and normal LTP. Brain Res Mol Brain Res. 2004;130:16-22.

29 Peters M, Mizuno K, Ris L, Angelo M, Godaux E, Giese KP. Loss of $\mathrm{Ca}^{2+} /$ calmodulin kinase kinase beta affects the formation of some, but not all, types of hippocampus-dependent long-term memory. J Neurosci. 2003;23:9752-9760.

30 Mizuno K, Peters M, Giese KP. The role of $\mathrm{Ca}^{2+} /$ calmodulindependent protein kinase kinases in learning and memory. Soc Neuroscience. 2003;Abstract No. 836.1. 
31 Richmond MA, Yee BK, Pouzet B, Veenman L, Rawlins JN, Feldon $\mathrm{J}$, et al. Dissociating context and space within the hippocampus: effects of complete, dorsal, and ventral excitotoxic hippocampal lesions on conditioned freezing and spatial learning. Behav Neurosci. 1999;113:1189-1203.

32 Moser E, Moser MB, Andersen P. Spatial learning impairment parallels the magnitude of dorsal hippocampal lesions, but is hardly present following ventral lesions. J Neurosci. 1993;13:3916-3925.

33 Balschun D, Wolfer DP, Gass P, Mantamadiotis T, Welzl H, Schutz G, et al. Does cAMP response element-binding protein have a pivotal role in hippocampal synaptic plasticity and hippocampus-dependent memory? J Neurosci. 2003;23:63046314.

34 Korzus E, Rosenfeld MG, Mayford M. CBP histone acetyltransferase activity is a critical component of memory consolidation. Neuron. 2004;42:961-972.

35 Morozov A, Muzzio IA, Bourtchouladze R, Van-Strien N, Lapidus $\mathrm{K}$, Yin D, et al. Rap1 couples cAMP signaling to a distinct pool of p42/44MAPK regulating excitability, synaptic plasticity, learning, and memory. Neuron. 2003;39:309-325.

36 Otto C, Kovalchuk Y, Wolfer DP, Gass P, Martin M, Zuschratter
$\mathrm{W}$, et al. Impairment of mossy fiber long-term potentiation and associative learning in pituitary adenylate cyclase activating polypeptide type I receptor-deficient mice. J Neurosci. 2001;21:5520-5527.

37 Tao X, Finkbeiner S, Arnold DB, Shaywitz AJ, Greenberg ME. $\mathrm{Ca}^{2+}$ influx regulates BDNF transcription by a CREB family transcription factor-dependent mechanism. Neuron. 1998;20: 709-726.

38 Yamada K, Mizuno M, Nabeshima T. Role for brain-derived neurotrophic factor in learning and memory. Life Sci. 2002;70: 735-744.

39 Tyler WJ, Alonso M, Bramham CR, Pozzo-Miller LD. From acquisition to consolidation: on the role of brain-derived neurotrophic factor signaling in hippocampal-dependent learning. Learn Mem. 2002;9:224-237.

40 Gorski JA, Balogh SA, Wehner JM, Jones KR. Learning deficits in forebrain-restricted brain-derived neurotrophic factor mutant mice. Neuroscience. 2003;121:341-354.

41 Levenson JM, Choi S, Lee SY, Cao YA, Ahn HJ, Worley KC, et al. A bioinformatics analysis of memory consolidation reveals involvement of the transcription factor c-rel. J Neurosci. 2004;24:3933-3943. 